\title{
MONITORING OF RISK FACTORS IN FAMILY MEMBERS OF PATIENTS WITH BREAST CANCER
}

Joana Santos', Filipe Almeida', Giselda Bezerra'1, Wanuska Portugal'1

${ }^{1}$ Centro Universitário Brasileiro - Recife (PE), Brazil.

Optimize the monitoring of risk factors among family members of patients with breast cancer. A systematic review of pertinent scientific articles was performed. For such, the PubMed database was searched for articles published between 2014 and 2016 using the following keywords: breast cancer; risk factors, and family monitoring. Four articles were encountered and two were selected for the present review. The International Agency for Research on Cancer estimates an incidence of 2.5 million diagnoses of breast cancer in the year 2020, which is a 117\% increase from the 1.15 million diagnoses in 2002. Family monitoring with the analysis of risk factors among family members is one of the most effective ways to ensure an early diagnosis. The analysis of genetic factors, a family history of cancer and smoking, among other risk factors, can assist in the early diagnosis of the disease and increase the chances of curing the patient. During the initial risk assessment, the determination of the family history is a fundamental tool for outlining the family "pedigree". Breast cancer is the second more frequent type of cancer in the world and the main cause of death among women in Brazil (37\%). The monitoring of risk factors through the analysis of genetic factors, family history, and hormonal disorders is fundamental to the early diagnosis of this disease. Some studies suggest that not all women have the same risk of breast cancer, and that certain factors, called risk factors, increase the likelihood of developing breast cancer. The monitoring of risk factors among family members of patients with a history of breast cancer is a valuable tool for the early diagnosis of the disease. Further studies involving the analysis of genetic aspects are needed to broaden the evaluation of risk factors for breast cancer. 